\title{
Small Molecule Stabilization of PINK-1/PINK1 Improves
}

\section{Neurodegenerative Disease}

*These authors contributed equally to this work

"Address correspondence to Natalia V. Kirienko, kirienko@rice.edu

\section{Abstract}

Macroautophagic recycling of dysfunctional mitochondria, known as mitophagy, is essential for mitochondrial homeostasis and cell viability. Accumulation of defective mitochondria and impaired mitophagy have been widely implicated in many neurodegenerative diseases, and loss-of-function mutations of two regulators of mitophagy, PINK1 and Parkin, are amongst the most common causes of recessive Parkinson's disease. Activation of mitophagy via pharmacological treatments may be a feasible approach for combating neurodegeneration. In this effort, we screened $\sim 45,000$ small molecules for the

17 ability to activate mitophagy. A high-throughput, whole-organism, phenotypic screen was conducted by monitoring stabilization of PINK-1/PINK1, a key event in mitophagy activation, in a Caenorhabditis elegans strain carrying a Ppink-1::PINK-1::GFP reporter. We obtained eight hits that induced mitophagy, as evidenced by increased mitochondrial fragmentation and autophagosome formation. Several of the

21 compounds also reduced ATP production, oxygen consumption, mitochondrial mass, and/or

22 mitochondrial membrane potential. Importantly, we found that treatment with two compounds, which 
we named PS83 and PS106 (more commonly known as sertraline) reduced neurodegenerative disease mitochondrial turnover.

Keywords: mitophagy, PINK-1, high-throughput, high-content, compound screening, neurodegenerative diseases, paralysis

\section{Introduction}

Although they are often simplistically characterized as the "powerhouse of the cell", mitochondria of cells and organisms.

Unfortunately, cells have not many methods to repair mitochondrial damage. When damage is limited, mitochondria fuse, allowing their content to be mixed and sorted, segregating the damaged material (by an unknown mechanism) into low-quality mitochondria targeted for destruction via macroautophagic degradation (hereafter referred to as mitophagy) (5). The best-known pathway for

42 triggering mitophagy is the PINK1/Parkin pathway, both of whose namesake members have been linked 43 to Parkinson's disease (5-7). Activation of this pathway begins with the PTEN-induced kinase 1 (PINK1), a 44 serine/threonine kinase that is constitutively expressed and targeted to mitochondria (5). Upon arrival, 45 healthy mitochondria will import the kinase and it will be destroyed by matrix-resident proteases. If 
47 itself, activating its ability to phosphorylate other substrates, including the E3 ubiquitin ligase Parkin. Once

48 phosphorylated, Parkin will begin to ubiquitinate its targets, including outer mitochondrial membrane

49 proteins, which allows their recognition by the machinery that recruits the isolation membrane and begins

50 the process of engulfing mitochondria into an autophagosome. Once the autophagosome has closed, it

51 will fuse with lysosomes to develop autophagolysosomes, where the contents will start to be degraded.

Mitochondrial turnover is activated under certain physiological conditions, such as during the

maturation of erythrocytes (8), but evidence suggests that mitophagy participates in a broad variety of physiological functions, including in response to hypoxia and pathogen exposure (9-13). Additionally, mitophagy is surprisingly important for the maintenance of normal function for neurological cells. For example, mutations in both PINK1 and Parkin are linked with early-onset Parkinsonism $(6,14)$. Other types of mitochondrial dysfunction have been implicated in other neurodegenerative diseases (NDD), including

58 Huntington's disease, Alzheimer's, and multiple sclerosis (7, 15-18), indicating failed mitochondrial maintenance as a common feature of these diseases.

Importantly, there is credible evidence that stimulating mitophagy can help mitigate some aspects of these disorders. For example, overexpression of PINK1 can restore mitophagy in a Drosophila model of Huntington's disease and reduce disease symptoms (16). However, the consensus opinion is that treating most of these disorders will be more effective using small molecule therapies, rather than genetic modifications (19). Promising evidence in this field area exists as well. In an elegant recent study, Fang and colleagues demonstrated that a number of molecules, including urolithin $A$, could stimulate mitophagy and reduce disease burden in Caenorhabditis elegans, human neuronal cells, and two murine Alzheimer's disease models (20). Additional efforts in this area have also been reviewed recently (19). 
71 and high-throughput compounds screening due to its small size, short generation and lifespan, and the

72 ability to simultaneously counterscreen for toxic compounds (24). Finally, as C. elegans is transparent,

73 intact worms can be used for high-content screens where more nuanced or complex phenotypes can be

74 used.

75 In this study, we used a high-throughput, high-content phenotypic screen using C. elegans carrying GFP tagged full-length PINK-1/PINK1 (Ppink-1::PINK-1::GFP) to identify a panel of small molecules that stabilize the kinase and verified their potentials in the activation of mitophagy. Four of the eight tested PS molecules significantly reduced mitochondrial membrane potential, while two of the others reduced mitochondrial mass loss. Neuroprotective properties of the selected PS compounds were tested in a transgenic $C$. elegans model of Alzheimer's disease, where we discovered that two of the compounds significantly delayed paralysis in a PINK-1/PINK1-dependent manner. One of the two compounds was also able to reduce aggregate formation in a C. elegans polyglutamine-protein aggregation model. The compounds generally showed relatively low toxicity to human astroglial and prostate epithelial cells, increasing their promise.

\section{Results}

(Fig. 1a). To identify small molecules that promote PINK1 stability, we leveraged a C. elegans strain 
Worms were exposed to the negative control or to sodium selenite for $24 \mathrm{~h}$ and then GFP was

We determined the $Z^{\prime}$-factor of the assay to be 0.52 (Fig. 1c). $Z^{\prime}$-factors range from - $\infty$ to 1 , with the 0.5 indicates a strong ability to detect even moderately weak hits. type worms (which do not express GFP) to eliminate the possibility that the compounds themselves were

(Fig S1, Table S1). The final hit rate was $0.018 \%$, which is somewhat lower than is commonly found from

112 to explore broad amounts of chemical space. 
DMSO mostly showed this state (>85\%, Fig. 2a)

Qualitative analysis of mitochondrial network structure showed that treatment with the PS compounds significantly increased the tendency towards intermediate (partially fragmented) or punctate (strongly fragmented) mitochondrial network. Four of the compounds, PS30, PS34, PS127, and PS143, induced substantial fragmentation, as shown by the dissolution of the tubular network into discrete punctae. PS83, PS103, PS106, and PS135 induced a milder disruption of the mitochondrial network, although each was statistically significant. Disruption of mitochondria fission-fusion homeostasis is consistent with existing evidence that mitochondrial fragmentation is necessary for mitophagy to occur $(10,37)$.

To confirm the formation of autophagosomes, we exposed a worm strain expressing mCherry::LGG-1 to the PS compounds. Under normal conditions, LGG-1, and its mammalian ortholog MAP1LC3, exhibit a diffuse cytoplasmic localization (38). During the formation of the isolation membrane (which will become the autophagosome), MAP1LC3 is crosslinked to the lipids that will comprise this membrane, converting the localization from a diffuse pattern into bright punctae $(38,39)$. Punctae were qualitatively assessed as few, medium, or many, with representative images shown (Fig. 2b). Compared to the DMSO control, several of the PS compounds increased punctae localization of mCherry::LGG-1/LC3, including two strong hits (PS30 and PS106) and three weak hits (PS83, PS103, and PS135), indicating increased autophagosomal formation. Intriguingly, three compounds (PS34, PS127, and PS143) reduced the production of autophagosomes, an unexpected outcome from stimulating the accumulation of PINK-1/PINK1. Two mitochondrial disruptors, rotenone (which blocks Complex I of the electron transport chain (ETC)) and carbonyl cyanide $m$-chlorophenyl hydrazine (CCCP, a proton uncoupler that dissipates the electrochemical gradient on the mitochondrial membrane), were used to validate the assay.

After formation of the autophagosome is completed, the next step in mitophagy is for the autophagosome to fuse with lysosomes to form autophagolysosomes (40). To observe this process, 
worms were treated with PS compounds, CCCP, rotenone, or vehicle control for $15 \mathrm{~h}$. Afterward, worms

144 were stained with LysoTracker Red DND-99, a dye that specifically labels acidic cellular compartments.

145 This dye is routinely used to label lysosomes and autophagolysosomes $(41,42)$. Uptake was quantitatively

measured by using flow vermimetry (43). Increased LysoTracker Red fluorescence was seen after

147 treatment with PS34, PS127, PS143, and CCCP, but not with the rest of the PS compounds (Fig. 2c) (44).

148 In contrast, treatment with PS30, PS83, PS103, PS106, or PS135 fragmented mitochondria but did not

149 result in substantial formation of acidified organelles. This outcome was also seen for rotenone, which

150 triggered the early stages of mitophagy (i.e., PINK-1/PINK1 stabilization, formation of the isolation

151 membrane and autophagosome) but precluded acidification of the autophagosomes, preventing

152 increased fluorescence from LysoTracker Red. This is consistent with reports elsewhere regarding the

153 accumulation of autophagosomes and decreased autophagic completion after rotenone exposure (45,

154 46). This failure has been attributed to ATP depletion, which prevents the lysosomal vacuolar ATPase from consuming ATP to acidify the autophagolysosome (45).

Worms carrying a ubiquitously expressed firefly luciferase to provide real-time readout of ATP (47, fragmentation (PS30, PS34, PS127, and PS143, Fig. 2a) also caused significant drop in ATP production (Fig. autophagolysosomes.

To investigate on whether ATP production failure was due to the inhibition of the ETC, we monitored

162 the last stage of the chain by measuring oxygen consumption rate (OCR) (49). Only PS34, PS127, or 163 rotenone significantly lowered oxygen consumption rate (with PS127 completely abolishing respiration) 164 (Fig. 3a). 
To identify the mechanisms giving rise to mitophagy after compound treatment, several mitochondrial phenotypes were assayed. First, mitochondria were stained with MitoTracker Red, which accumulates in mitochondria proportionally to their membrane potential. Nonyl-acridine orange, a dye that binds to cardiolipin and is comparatively insensitive to mitochondrial membrane potential $(50,51)$,

171 was used as a proxy to measure mitochondrial mass. CCCP, which dissipates mitochondrial membrane 172 potential (52), was used as a control (Fig. 3b). CCCP reduced both mitochondrial mass and mitochondrial 173 membrane potential, which is consistent with uncoupler treatment.

174 Significant reduction of mitochondrial membrane potential was observed in worms treated with PS30,

175 PS103, PS106, and PS135 (Fig. 3b). This reduction did not appear to be accompanied by corresponding

176 decrease in mitochondrial mass, may be indicative of weak uncoupling activity. These data suggest that

177 for at least four compounds, the loss of mitochondrial membrane potential might be the trigger for 178 mitophagy activation. In contrast, treatment with PS34 or PS127 reduced apparent mitochondrial mass, 179 but not mitochondrial membrane potential, which may indicate that there are fewer mitochondria with 180 increased membrane potential. We previously observed similar changes when worms' diet was 181 supplemented with vitamin B12, which improved mitochondrial health (53). Neither PS83 nor PS143 182 significantly affected mitochondrial membrane potential or mass (Fig. 3 b). Combined, these results 183 suggest that the loss of ATP content in PS30 and PS135 was not due to failure of the mitochondrial ETC, 184 as in the case for PS34 and PS127. Instead, PS30, PS103, and PS135, and PS106 to a lesser extent, may 185 have mild uncoupling activity.

186 Another common reason for the induction of mitophagy is the accumulation of ROS $(54,55)$. To test 187 whether the PS compounds induced ROS production, worms were treated with compounds for $15 \mathrm{~h}$, and 188 then were stained with dihydroethidium, a non-fluorescent, redox-sensitive dye that is converted to 189 fluorescent 2-hydroxyethidium by reaction with superoxide (56). Surprisingly, none of the compounds 190 appeared to significantly increase ROS production (Fig. 3c). The positive control, CCCP, validated that the 
assay was being performed correctly (Fig. 3c).

Autophagic activation integrates a large number of signals, including stress and nutrient status. For compounds. Four compounds (PS34, PS83, PS127, and PS143) triggered DAF-16 translocation into the nucleus, demonstrating that it has been activated (Fig. 4a). The same four compounds also activated the commonly used to confirm SKN-1 activation, albeit to a lower level than DAF-16 (Fig. 4b). was exposed to the PS compounds or to RPW-24, a positive control. Only three of the compounds, PS30, PS135, and PS143, induced PMK-1 pathway activity (Fig. 4c). 
215

216

217

218

219

220

221

222

223

224

225

226

227

228

concentrations (Fig. S2). Two compounds, PS83 and PS106, substantially reduced paralysis at $5 \mu \mathrm{M}$ and $25 \mu \mathrm{M}$, respectively, and were comparable to the positive control metformin (Fig. 5a-c and Fig. S2). Another compound, PS103, provided a more modest, but still significant decrease in paralysis at $25 \mu \mathrm{M}$

(Fig. S2). Alternatively, it is possible that PS83- and PS106-mediated rescue was an artifact and was independent of PINK-1/PINK1 stabilization. To test this, RNAi was used to knock down pink-1 expression in the $\beta$-amyloid-expressing strain prior to compound exposure. Consistent with our interpretation, pink1(RNAi) completely removed the ability of PS83 or PS106 to delay paralysis, and made worms treated with PS83 or PS106 indistinguishable from vehicle controls (Fig. $\mathbf{5 d , ~ e ) . ~}$

To test whether the compounds had a broad effect on NDD models, we obtained a worm strain that expresses a YFP-tagged protein with an engineered polyglutamine (polyQ) repeat of 82 consecutive glutamine residues (Q82::YFP) (65). PolyQ repeats are causative for at least ten different neurodegenerative diseases, with the best-known being Huntington's chorea (66). Expression of the chimeric Q82::YFP product under the control of a tissue-specific promoter (e.g., unc-54 or vha-6) causes Q82::YFP aggregation in the target tissue (65).

Young adult worms expressing the Q82::YFP construct under the intestinal promoter vha-6 were treated with PS83 $(5 \mu \mathrm{M})$ or PS106 $(25 \mu \mathrm{M})$ for $24 \mathrm{~h}$, and then aggregates were manually counted under low magnification (Fig. 6). We found that PS83 showed no clear difference from vehicle alone (Fig. 6a), while PS106 treatment significantly reduced the number of aggregates (Fig. 6b). Previously, the Morimoto lab implicated activated DAF-16/FOXO in limiting Q82 aggregation and paralysis when aggregates form in body wall muscles (65). Since PS106 did not induce DAF-16 nuclear localization, the precise regulatory pathway induced by PS106 and its role in providing neuroprotection will need to be further elucidated.

\section{PS compound exposure shows limited toxicity}

While activation of mitophagy can provide a means to overcome some aspects of NDD, overactivation 
of mitophagy may lead to excess mitochondrial loss, bioenergetic deficits, and cellular death (67). To test whether prolonged exposure to PS compounds causes death, survival of $C$. elegans and human cells was measured. For C. elegans, worms were exposed to the eight PS compounds at four different concentrations for $72 \mathrm{~h}$ and then were incubated with Sytox Orange, a cell-impermeant dye that stains DNA in dead worms. Treatment with most compounds showed greater than $75 \%$ of survival (as normalized to the DMSO control) at concentrations of $25 \mu \mathrm{M}$ or less (Fig. 7a). In all cases, worms survived the lowest tested dosage; in all but one (i.e., PS83), the highest dose caused at least partial death (Fig. 7a). This suggests that some optimization would be necessary to see protective effects. Importantly, the two compounds that reduced paralysis rate in the $C$. elegans Alzheimer's model (PS83 and PS106) did not impair survival at tested concentrations of up to $100 \mu \mathrm{M}$.

Cytotoxicity of chronic, $72 \mathrm{~h}$ exposure to PS compounds at similar concentrations was also measured in two human cell lines, SVG-P12 (an astroglial cell line) and RWPE-1 (prostate epithelial cells) (Fig. 7b, c). Differential Hoechst / propidium iodide staining was used to assess cell death. Exposure to either PS103 or PS106 at concentrations higher than $10 \mu \mathrm{M}$ showed substantial toxicity in both cell lines. Interestingly, PS34, PS127, PS135, and PS143 were less toxic to human cells than to C. elegans.

\section{Discussion}

Stimulation of mitophagy has proven to be a promising therapeutic target for neurodegenerative diseases (68) and may be beneficial for healthy aging. Using a high-throughput, high-content phenotypic screen, we obtained and characterized eight PINK-1/PINK1-stabilizing compounds. Interestingly, two of the compounds had previously been associated with alterations in mitochondrial function.

PS103, commonly known as triclosan or irgasan, has been linked with a variety of mitochondrial dysfunction, including uncoupling of the mitochondrial membrane potential by reversible protonation of the phenoxy group (69-71) and inhibition of Complex II of the ETC (72). Triclosan has also been associated 
with increased mitochondrial ROS, reduced mitochondrial mass, and disruptions in mitochondrial morphology (73). The ability of triclosan to cause several types of mitochondrial damage, apparently with different proximal factors (72), somewhat reduces its value as a therapeutic agent.

Despite its common appearance in a wide variety of consumer products, questions about the safety of triclosan remain, even if used externally. For example, in addition to its role in mitochondrial disruption, triclosan also has the potential to disrupt endocrine function, affect immunity, disrupt calcium and zinc homeostasis, and alter lipid metabolism (74). Triclosan also has a strong potential for bioaccumulation (75), which is undesirable in a maintenance medicine. Given these caveats, the potential for triclosan to be developed into a treatment for NDD seems small.

The potential for PS106, more commonly known as sertraline, is substantially greater. Sertraline is thought to bind to the serotonin transporter (SERT) in the presynaptic neuron, preventing reabsorption of serotonin and prolonging synaptic signaling. Sertraline is a well-known compound with carefully studied pharmacological effects and is one of the most commonly prescribed psychiatric medications in the US. Our data contribute to an ongoing discussion about the potential for sertraline as a treatment for one or more NDDs.

Probably the earliest hint that sertraline may have some unexpected effect on mitochondria came in a report attempting to identify 'hidden' drug targets, where the authors determined that sertraline had several characteristics similar to the well-known mitochondrial toxin rotenone (76). Not long after, Kumar and colleagues demonstrated that sertraline treatment could ameliorate damage caused by the mitotoxic agent 3-nitropropionic acid (77). Recently, it was demonstrated that sertraline prevents the function of the mitochondrial VDAC1, reducing cellular ATP, increasing the ADP/ATP ratio, and activating autophagy through mTOR (78).

Sertraline increases survival and neurogenesis at pharmacologically relevant concentrations in several murine models of Huntington's $(79,80)$ and physiological outcomes (e.g., grip strength, coordination, 
locomotor activity, etc.) in rat models of Huntington's and Parkinson's diseases (81, 82). Given the

frequent co-occurrence of depression with NDD, it is not surprising that sertraline is often prescribed to these patients suffering from these disorders. Promisingly, some Parkinson's patients receiving sertraline have shown improvement in their symptoms $(83,84)$. It is clear, given these findings, that a more systematic study of the potential for sertraline for the treatment of NDD is warranted. several similarities in treatment outcome to sertraline and triclosan. For example, all three compounds caused relatively minor mitochondrial fragmentation, but little other effect. PS103 and PS106 caused greater depolarization, while PS83 apparently did not. Searching the literature for other reports of PS83 failed to provide additional clues. For the time being, we can only conclude that it stabilizes PINK-1::GFP and that this stabilization does not appear to be a consequence of depolarizing the mitochondrial membrane or disrupting the electron transport chain.

Two of the compounds, PS30 and PS135, appear likely to be mitochondrial uncouplers. They reduced mitochondrial membrane potential and decreased ATP content, but oxygen respiration continued unabated. Like PS83, relatively little is known about PS30 or PS135. However, an analog of PS30, known as SMTC1100, has been shown to be helpful in Duchenne muscular dystrophy (85). This fatal, progressive disorder is characterized by wasting muscle loss due to disruption of the dystrophin protein, which leads to mitochondrial dysfunction $(86,87)$. This suggests that a larger portion of the scaffold may have a positive effect on mitochondrial recycling in chronic degenerative disorders. 
311 potential, suggesting that they disrupt degradation of PINK-1/PINK1 in a different fashion. Interestingly,

312 they were also amongst the strongest activators of GST-4/Nrf and DAF-16/FOXO, which may indicate that

313 they are causing other damage to the cells.

314 The accumulation of mitochondrial damage, and concomitant degradation of function, is associated

315 with both aging and neurodegenerative disease. Mitophagy also appears to be inherently limited in

316 mature neurons (reviewed in (68)), which may explain why this tissue is more sensitive to mitochondrial

317 damage in the first place. Increasingly, it has been hypothesized and demonstrated that increasing

318 mitophagy in these cells may promote better cellular health and aging (reviewed in (68)).

319 Unfortunately, a relative dearth of compounds appropriate for this purpose is currently available, and

320 identification of new compounds requires a relatively complex screening process, like the whole-organism

321 phenotypic approach demonstrated herein. Although the eight compounds we identified and studied

322 have considerable promise (especially sertraline), substantial additional study is needed to further

323 understand their effects.

325 Methods

\section{C. elegans strains and maintenance}

Worms were synchronized by hypochlorite isolation of eggs from gravid adults, followed by hatching of eggs in S Basal. 6,000 synchronized L1 larvae were transferred onto $10 \mathrm{~cm}$ standard nematode growth medium (NGM) plates seeded with Escherichia coli strain OP50 as a food source (88). After transfer, worms were grown at $20^{\circ} \mathrm{C}$ for 50 hours prior to experiments, or for three days for the next eggs isolation.

331 Young adult worms were used for all assays unless otherwise noted. Strains used in this study include: N2 332 Bristol (wild-type), NVK90 |pink-1(tm1779); houls001 \{byEx655 [Ppink-1::PINK-1::GFP + Pmyo- 
4::GFP::NLS]\} (91), AY101 \{acls101 [Pirg-5::GFP + rol-6(su1006)]\} (92), PE255 ffels5 [Psur-

5::Iuciferase::GFP + rol-6(su1006)]\}, GMC101 \{dvls100 [Punc-54::A-beta-1-42::unc-54 3'-UTR + Pmtl-

\section{Bacterial strains}

Bacterial strains used in this study included E. coli OP50, RNAi-competent OP50 (xu363), and RNAicompetent E. coli HT115 (obtained from the Ahringer RNAi library). Plasmids were isolated, purified, and sequenced prior to transformation into RNAi-competent OP50 (xu363). Transformed bacteria were confirmed to contain the plasmid of interest by sequencing as well.

\section{RNA interference protocol}

346 RNAi-expressing bacteria were cultured and seeded onto NGM plates supplemented with $25 \mu \mathrm{g} / \mathrm{mL}$

347 carbenicillin and $1 \mathrm{mM}$ IPTG. For double RNAi, bacterial cultures expressing either vector(RNAi) or pink1(RNAi) were mixed with sterility-inducing $c d c-25.1$ (RNAi) with a 1:1 ratio. For experiments with GMC101

349 strain, 2,000 synchronized L1 larvae were plated onto $6 \mathrm{~cm}$ RNAi plates and grown at $20^{\circ} \mathrm{C}$ for 48 hours

350 prior to use for experiments. For experiments with GF66 strain, 50 gravid hermaphrodites were

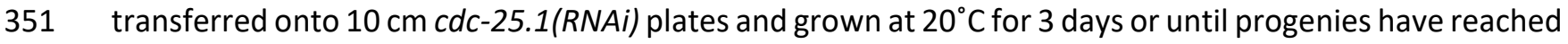
352 the L4 stage. Sterile progenies were then transferred to treatment plates for the next experimental step.

\section{Statistical Analysis}

355 RStudio (version 3.6.3) was used to perform statistical analysis. Chi-square test was used to calculate the 356 significance between qualitative variables. One-way analysis of variance (ANOVA) was performed to 357 calculate the significance of a treatment when there were three or more groups in the experimental setting. To follow, Dunnett's test (R package DescTools, version 0.99.34) was performed to calculate 
statistical significance or $p$ values between each group of the statistically significant experimental results.

Student's $t$-test analysis was performed to calculate the $p$ values when comparing two groups in an replicates were performed.

\section{C. elegans library screening and compound exposure assays}

366 Synchronized sterile young adult worms were washed from NGM plates seeded with OP50 into a conical tube and rinsed three times. Worms were then sorted into a 384-well plate ( 25 worms/well) for the initial screen or a 96-well plate ( 100 worms/well, half-area) for all other assays. For the initial highthroughput screen, each well in the 384-well plates contained $50 \mu \mathrm{M}$ of compounds, and the exposure

370 length was $24 \mathrm{~h}$. For other compound/chemical exposure assays, S Basal supplemented with $50 \mu \mathrm{M}$ PS 371 compounds, $7 \mathrm{mM}$ sodium selenite (Alfa Aesar), $10 \mu \mathrm{M}$ CCCP (Sigma), $50 \mu \mathrm{M}$ rotenone (Sigma), $50 \mu \mathrm{M}$

372 juglone (Sigma), $100 \mu \mathrm{M}$ RPW-24, or DMSO (solvent control) was added into the wells of the 96-well plate 373 to a final volume of $100 \mu \mathrm{L}$. Worms were imaged with Cytation5 Cell Imaging Multi-Mode Reader (BioTek 374 Instruments) every day for three days for the initial screen or every two hours for twenty four hours for 375 the other assays.

\section{Imaging and fluorescence quantification}

378 For visualization of the worm reporter strains NVK90, TJ356, CL2166, AY101, and GF66, Cytation5 379 automated microscope was used. All imaging experiments were performed with identical settings within 380 three biological replicates of each strain. GFP quantifications were performed by using Gen5 3.10 software 381 and CellProfiler. For visualization of the worm reporter strains SJ4103 and VK1241, worms were 382 immobilized by using $1 \mathrm{mM}$ levamisole and then transferred onto 3\% agarose pad. Worms were imaged 
using fluorescence microscope (Zeiss ApoTome.2 Imager.M2, Carl Zeiss, Germany) with a 63x (SJ4103) or 40x (VK1241) objective magnification.

\section{Fluorescent-dye staining and quantification}

387 Approximately $400 \mathrm{~N} 2$ worms (in four wells, each containing 100 worms) were treated with PS compounds or corresponding controls for $15 \mathrm{~h}$ in 96 -well plate. At $14 \mathrm{~h}$ of incubation, fluorescent dye with a final concentration of $10 \mu \mathrm{M}$ for LysoTracker Red, $10 \mu \mathrm{M}$ for NAO, $4.375 \mu \mathrm{M}$ for MitoTracker Red, or 3 $\mu \mathrm{M}$ for $\mathrm{DHE}$, were added. For background subtraction, $\mathrm{S}$ Basal without any dye was added. Worms were washed three times to remove any remaining compounds or dye before fluorescence measurement was taken via flow vermimetry (COPAS Biosort, Union Biometrica).

\section{ATP production measurement}

A worm strain carrying firefly luciferase gene followed by GFP (PE255) was used for ATP production out according to the published protocol (94). Essentially, at $18 \mathrm{~h}$ of incubation, worms were washed three times to remove any remaining compounds. Luminescence buffer was then added, incubated for 3 minutes, and fluorescence (485/20 excitation and 528/20 emission) and luminescence were measured with Cytation5 (BioTek Instruments).

401

\section{Oxygen consumption rate measurement}

4033,000 N2 worms were sorted into each well of a 6-well plate. PS compounds, vehicle control DMSO, or 404 positive control rotenone, and E. coli OP50 (final OD 600 : 0.05 ) were then added into each well to a final concentration of $50 \mu \mathrm{M}$. Two wells for each compound were used, totaling to 6,000 worms per condition. 
407

408

409

410

411

412

413

414

415

416

417

418

419

420

421

422

423

424

425

426 427 of the worms.

428

429

430 the plate.

three times to remove residual compounds. Oxygen consumption was measured by using a biological oxygen monitor (YSI 5300) and a Clark-type oxygen electrode (YSI 5301) (Yellow Springs Instrument) at $20^{\circ} \mathrm{C}$ as previously described (49). Oxygen consumption was recorded continuously for ten minutes.

\section{Beta-amyloid-induced paralysis and scoring}

Synchronous L1 population of C. elegans strain GMC101 (95) was reared on E. coli OP50 at $20^{\circ} \mathrm{C}$ for 48 hours to reach the L4 stage. 60 worms were then transferred onto each $35 \mathrm{~mm}$ NGM plates containing $250 \mu \mathrm{M}$ 5-fluoro-2'-deoxyuridine (FUDR) and PS compounds (see Fig. S2 for final concentrations) or corresponding DMSO control. Worms were then incubated at $25^{\circ} \mathrm{C}$ to induce paralysis and scored every day for five days. Paralysis was indicated by inability to complete a full sigmoidal body movement spontaneously or following stimulation with a pick. Paralyzed worms were counted and removed from

\section{Polyglutamine protein aggregation scoring}

50 gravid hermaphrodites of C. elegans strain GF66 expressing polyglutamine (Pvha-6::Q82::YFP) (65) were reared on E. coli OP50 expressing $c d c-25.1$ (RNAi) and grown at $20^{\circ} \mathrm{C}$ for 3 days or until progenies have reached the L4 stage. L4 worms were then transferred onto treatment plates as for the betaamyloid-induced paralysis assay, but kept at $20^{\circ} \mathrm{C}$. After 24 hours, worms were transferred into a 96-well plate, washed three times, immobilized with $1 \mathrm{mM}$ levamisole, and imaged with Cytation 5 automated microscope with a $4 x$ objective magnification. The number of aggregates were counted manually for each

\section{C. elegans compound toxicity assay}

25 synchronized SS104 (g/p-4) young adult worms were sorted into 384-well plate. Compounds (final 
431

432

433

434

435

436 incubation mixtures or solvent-control wells never exceeded $0.5 \%(\mathrm{v} / \mathrm{v})$.

453 microscope. CellProfiler software was used to quantify worms' death.

\section{Mammalian cell culture and compound toxicity assay} concentration. Gen5 3.10 software were used for imaging and cell counting pipeline.

concentration: $100 \mu \mathrm{M}, 50 \mu \mathrm{M}, 25 \mu \mathrm{M}$, and $12.5 \mu \mathrm{M}$ ) were mixed with E. coli OP50 as food source (final $\left.\mathrm{OD}_{600}: 0.05\right)$, and then added into each well. Plates were incubated at $25^{\circ} \mathrm{C}$ for 72 hours. Plates were washed three times and worms were stained with SYTOX ${ }^{\mathrm{TM}}$ Orange nucleic acid dye to stain dead worms. After $14 \mathrm{~h}$ of incubation with SYTOX ${ }^{\mathrm{TM}}$, plates were washed and imaged with Cytation5 automated

Human fetal glial cells (SVGp12) and prostate epithelial cells (RWPE-1) were purchased from ATCC (Manassas, VA, USA). SVGp12 cells were cultured in minimum essential Eagle medium (ThermoFisher) containing 10\% FBS (fetal bovine serum; Corning, Manassas, VA, USA) and RWPE-1 cells were cultured in Defined Keratinocyte SFM (ThermoFisher) with growth supplement at $37^{\circ} \mathrm{C}$ in a humidified $5 \% \mathrm{CO}_{2}$ atmosphere. The solution of penicillin-streptomycin (Gibco, Gaithersburg, MD, USA) was used at $1 \%$ final

Fluorescent cell labeling with Hoechst 33342 (ThermoFisher) and propidium iodide (ThermoFisher) with subsequent automated cell counting was used as cytotoxicity assay as previously described (96). Cytation5 Cell Imaging Multi-Mode Reader with DAPI and Texas Red filter sets (BioTek Instruments) and

For each cytotoxicity experiment, cells were seeded at a density of $10^{3}$ cells/well in 96-well plates and cultured for $24 \mathrm{~h}$ prior to the drug treatment. Cells were then treated with PS compounds or DMSO (solvent control) at specified concentrations (see Fig. 7) for $72 \mathrm{~h}$ in $100 \mu \mathrm{L}$ of complete media. All viability rates were normalized to the corresponding solvent-control wells. The DMSO concentrations in the

Acknowledgements: $C$. elegans strains used were obtained from the CGC. We thank Daniel Kirienko for 
comments on the manuscript and Maria Hancu for technical assistance.

456

Competing interests: The authors have declared that no competing interests exist.

\section{References}

1. Weinberg Samuel E, Sena Laura A, Chandel Navdeep S. Mitochondria in the Regulation of Innate and Adaptive Immunity. Immunity. 2015;42(3):406-17.

2. Newmeyer D, Ferguson-Miller S. Mitochondria: releasing power for life and unleashing the machineries of death. Cell. 2003;112(4):481-90.

3. Pinton P, Giorgi C, Siviero R, Zecchini E, Rizzuto R. Calcium and apoptosis: ER-mitochondria Ca2+ transfer in the control of apoptosis. Oncogene. 2008;27(50):6407-18.

4. Tait S, Green D. Mitochondria and cell signalling. Journal of cell science. 2012;125(Pt 4):807-15.

5. Youle RJ, Narendra DP. Mechanisms of mitophagy. Nat Rev Mol Cell Biol. 2011;12(1):9-14.

6. Song S, Jang S, Park J, Bang S, Choi S, Kwon KY, et al. Characterization of PINK1 (PTEN-induced putative kinase 1) mutations associated with Parkinson disease in mammalian cells and Drosophila. J Biol Chem. 2013;288(8):5660-72.

7. Martín-Maestro P, Gargini R, Perry G, Avila J, García-Escudero V. PARK2 enhancement is able to compensate mitophagy alterations found in sporadic Alzheimer's disease. Hum Mol Genet. 2016;25(4):792-806.

8. Sandoval H, Thiagarajan P, Dasgupta S, Schumacher A, Prchal J, Chen M, et al. Essential role for Nix in autophagic maturation of erythroid cells. Nature. 2008;454(7201):232-5.

9. Kang $D$, Kirienko DR, Webster $P$, Fisher AL, Kirienko NV. Pyoverdine, a siderophore from Pseudomonas aeruginosa, translocates into $\mathrm{C}$. elegans, removes iron, and activates a distinct host response. Virulence. 2018;9(1):804-17.

10. Kirienko NV, Ausubel FM, Ruvkun G. Mitophagy confers resistance to siderophore-mediated killing by Pseudomonas aeruginosa. Proceedings of the National Academy of Sciences. 2015;112(6):18216.

11. Wu H, Chen Q. Hypoxia activation of mitophagy and its role in disease pathogenesis. Antioxidants \& redox signaling. 2015;22(12):1032-46.

12. Boya P, González-Polo RA, Casares N, Perfettini JL, Dessen P, Larochette N, et al. Inhibition of macroautophagy triggers apoptosis. Mol Cell Biol. 2005;25(3):1025-40.

13. Lemasters JJ. Selective Mitochondrial Autophagy, or Mitophagy, as a Targeted Defense Against Oxidative Stress, Mitochondrial Dysfunction, and Aging. Rejuvenation Research. 2005;8(1):3-5.

14. Burchell VS, Nelson DE, Sanchez-Martinez A, Delgado-Camprubi M, Ivatt RM, Pogson JH, et al. The Parkinson's disease-linked proteins Fbxo7 and Parkin interact to mediate mitophagy. Nat Neurosci. 2013;16(9):1257-65.

15. Wong YC, Holzbaur EL. The regulation of autophagosome dynamics by huntingtin and HAP1 is disrupted by expression of mutant huntingtin, leading to defective cargo degradation. J Neurosci. 2014;34(4):1293-305.

16. Khalil B, El Fissi N, Aouane A, Cabirol-Pol MJ, Rival T, Lievens JC. PINK1-induced mitophagy promotes neuroprotection in Huntington's disease. Cell Death Dis. 2015;6:e1617.

17. Ebrahimi-Fakhari D, Saffari A, Wahlster L, Di Nardo A, Turner D, Lewis TL, et al. Impaired Mitochondrial Dynamics and Mitophagy in Neuronal Models of Tuberous Sclerosis Complex. Cell Rep. 2016;17(4):1053-70. 
18. Wu M, Liu X, Chi X, Zhang L, Xiong W, Chiang SMV, et al. Mitophagy in Refractory Temporal Lobe Epilepsy Patients with Hippocampal Sclerosis. Cell Mol Neurobiol. 2018;38(2):479-86.

19. Lou G, Palikaras K, Lautrup S, Scheibye-Knudsen M, Tavernarakis N, Fang EF. Mitophagy and Neuroprotection. Trends Mol Med. 2020;26(1):8-20.

20. Fang EF, Hou Y, Palikaras K, Adriaanse BA, Kerr JS, Yang B, et al. Mitophagy inhibits amyloid-beta and tau pathology and reverses cognitive deficits in models of Alzheimer's disease. Nat Neurosci. 2019;22(3):401-12.

21. Link C. Expression of human beta-amyloid peptide in transgenic Caenorhabditis elegans. Proceedings of the National Academy of Sciences of the United States of America. 1995;92(20):9368-72.

22. Nass R, Miller D, Blakely R. C. elegans: a novel pharmacogenetic model to study Parkinson's disease. Parkinsonism \& related disorders. 2001;7(3):185-91.

23. Satyal S, Schmid tE, Kitagawa K, Sondheimer N, Lindquist S, Kramer J, et al. Polyglutamine aggregates alter protein folding homeostasis in Caenorhabditis elegans. Proceedings of the National Academy of Sciences of the United States of America. 2000;97(11):5750-5.

24. Chen X, Barclay J, Burgoyne R, Morgan A. Using C. elegans to discover therapeutic compounds for ageing-associated neurodegenerative diseases. Chemistry Central journal. 2015;9(65).

25. Teschendorf $D$, Link $C$. What have worm models told us about the mechanisms of neuronal dysfunction in human neurodegenerative diseases? Molecular neurodegeneration. 2009;4(38).

26. Geisler S, Holmström K, Skujat D, Fiesel F, Rothfuss O, Kahle P, et al. PINK1/Parkin-mediated mitophagy is dependent on VDAC1 and p62/SQSTM1. Nature cell biology. 2010;12(2).

27. Lazarou M, Jin S, Kane L, Youle R. Role of PINK1 binding to the TOM complex and alternate intracellular membranes in recruitment and activation of the E3 ligase Parkin. Developmental cell. 2012;22(2).

28. Lazarou M, Sliter DA, Kane LA, Sarraf SA, Wang C, Burman JL, et al. The ubiquitin kinase PINK1 recruits autophagy receptors to induce mitophagy. Nature. 2015;524(7565):309-14.

29. Sämann J, Hegermann J, von Gromoff E, Eimer S, Baumeister R, Schmidt E. Caenorhabditits elegans LRK-1 and PINK-1 act antagonistically in stress response and neurite outgrowth. The Journal of biological chemistry. 2009;284(24).

30. Kim E, Choi K. A critical role of superoxide anion in selenite-induced mitophagic cell death. Autophagy. 2008;4(1):76-8.

31. Kim EH, Sohn S, Kwon HJ, Kim SU, Kim MJ, Lee SJ, et al. Sodium selenite induces superoxidemediated mitochondrial damage and subsequent autophagic cell death in malignant glioma cells. Cancer Res. 2007;67(13):6314-24.

32. Kamentsky L, Jones TR, Fraser A, Bray MA, Logan DJ, Madden KL, et al. Improved structure, function and compatibility for CellProfiler: modular high-throughput image analysis software. Bioinformatics. 2011;27(8):1179-80.

33. Anderson QL, Revtovich AV, Kirienko NV. A High-throughput, High-content, Liquid-based C. elegans Pathosystem. J Vis Exp. 2018(137).

34. Moy TI, Conery AL, Larkins-Ford J, Wu G, Mazitschek R, Casadei G, et al. High-throughput screen for novel antimicrobials using a whole animal infection model. ACS Chem Biol. 2009;4(7):527-33.

35. Kirienko DR, Revtovich AV, Kirienko NV. A High-Content, Phenotypic Screen Identifies Fluorouridine as an Inhibitor of Pyoverdine Biosynthesis and Pseudomonas aeruginosa Virulence. mSphere. 2016;1(4).

36. Bajusz D, Rácz A, Héberger K. Why is Tanimoto index an appropriate choice for fingerprint-based similarity calculations? Journal of cheminformatics. 2015;7.

37. Chan D. Fusion and fission: interlinked processes critical for mitochondrial health. Annual review of genetics. 2012;46:265-87. 
38. Gosai S, Kwak J, Luke C, Long O, King D, Kovatch K, et al. Automated high-content live animal drug screening using C. elegans expressing the aggregation prone serpin $\alpha 1$-antitrypsin Z. PloS one. 2010;5(11). 39. Meléndez A, Tallóczy Z, Seaman M, Eskelinen E, Hall D, Levine B. Autophagy genes are essential for dauer development and life-span extension in C. elegans. Science (New York, NY). 2003;301(5638):1387-91.

40. Klionsky D, Eskelinen E, Deretic V. Autophagosomes, phagosomes, autolysosomes, phagolysosomes, autophagolysosomes... wait, I'm confused. Autophagy. 2014;10(4):549-51.

41. Sun Y, Li M, Zhao D, Li X, Yang C, Wang X. Lysosome activity is modulated by multiple longevity pathways and is important for lifespan extension in C. elegans. eLife. 2020;9:e55745.

42. DeVorkin L, Gorski S. LysoTracker staining to aid in monitoring autophagy in Drosophila. Cold Spring Harbor protocols. 2014;2014(9):951-8.

43. Tjahjono E, Revtovich AV, Kirienko NV. Imaging and Fluorescence Quantification in Caenorhabditis elegans with Flow Vermimetry and Automated Microscopy. Bio-protocol. 2021;11(10):e4024.

44. Lin D, Huang $Y$, Ho C, Hung P, Hsu M, Wang $T$, et al. Oxidative Insults and Mitochondrial DNA Mutation Promote Enhanced Autophagy and Mitophagy Compromising Cell Viability in Pluripotent Cell Model of Mitochondrial Disease. Cells. 2019;8(1):65.

45. Mader B, Pivtoraiko V, Flippo H, Klocke B, Roth K, Mangieri L, et al. Rotenone inhibits autophagic flux prior to inducing cell death. ACS chemical neuroscience. 2012;3(12):1063-72.

46. Garcia-Garcia A, Anandhan A, Burns M, Chen H, Zhou Y, Franco R. Impairment of Atg5-dependent autophagic flux promotes paraquat- and $\mathrm{MPP}^{+}$-induced apoptosis but not rotenone or 6hydroxydopamine toxicity. Toxicological sciences : an official journal of the Society of Toxicology. 2013;136(1):166-82.

47. Lagido C, Pettitt J, Flett A, Glover L. Bridging the phenotypic gap: real-time assessment of mitochondrial function and metabolism of the nematode Caenorhabditis elegans. BMC physiology. 2008;8.

48. Luz AL, Lagido C, Hirschey MD, Meyer JN. In Vivo Determination of Mitochondrial Function Using Luciferase-Expressing <i>Caenorhabditis elegans $</ i>$ : Contribution of Oxidative Phosphorylation, Glycolysis, and Fatty Acid Oxidation to Toxicant-Induced Dysfunction: Luciferase Assay for Mitochondrial Function. In: Costa LG, Davila JC, Lawrence DA, Reed DJ, editors. Current Protocols in Toxicology. Hoboken, NJ, USA: John Wiley \& Sons, Inc.; 2016. p. 25.8.1-.8.2.

49. Braeckman B, Houthoofd K, De Vreese A, Vanfleteren J. Assaying metabolic activity in ageing Caenorhabditis elegans. Mechanisms of ageing and development. 2002;123(2-3):105-19.

50. Keij J, Bell-Prince C, Steinkamp J. Staining of mitochondrial membranes with 10-nonyl acridine orange, MitoFluor Green, and MitoTracker Green is affected by mitochondrial membrane potential altering drugs. Cytometry. 2000;39(3):203-10.

51. Mileykovskaya E, Dowhan W. Cardiolipin membrane domains in prokaryotes and eukaryotes. Biochimica et biophysica acta. 2009;1788(10):2084-91.

52. Narendra D, Tanaka A, Suen D-F, Youle RJ. Parkin is recruited selectively to impaired mitochondria and promotes their autophagy. The Journal of Cell Biology. 2008;183(5):795-803.

53. Revtovich AV, Lee R, Kirienko NV. Interplay between mitochondria and diet mediates pathogen and stress resistance in Caenorhabditis elegans. PLoS Genet. 2019;15(3):e1008011.

54. Wang Y, Nartiss Y, Steipe B, McQuibban G, Kim P. ROS-induced mitochondrial depolarization initiates PARK2/PARKIN-dependent mitochondrial degradation by autophagy. Autophagy. 2012;8(10).

55. Wei X, Qi Y, Zhang X, Gu X, Cai H, Yang J, et al. ROS act as an upstream signal to mediate cadmiuminduced mitophagy in mouse brain. Neurotoxicology. 2015;46.

56. Zhao H, Kalivendi S, Zhang H, Joseph J, Nithipatikom K, Vasquez-Vivar J, et al. Superoxide reacts with hydroethidine but forms a fluorescent product that is distinctly different from ethidium: potential 
implications in intracellular fluorescence detection of superoxide. Free Radic Biol Med. 2003;34(11):135968.

57. Henderson ST, Johnson TE. daf-16 integrates developmental and environmental inputs to mediate aging in the nematode Caenorhabditis elegans. Curr Biol. 2001;11(24):1975-80.

58. Wu C-W, Deonarine A, Przybysz A, Strange K, Choe KP. The Skp1 Homologs SKR-1/2 Are Required for the Caenorhabditis elegans SKN-1 Antioxidant/Detoxification Response Independently of p38 MAPK. PLOS Genetics. 2016;12(10):e1006361.

59. Kwon S, Kim EJE, Lee SV. Mitochondria-mediated defense mechanisms against pathogens in Caenorhabditis elegans. BMB Rep. 2018;51(6):274-9.

60. Pukkila-Worley R. Surveillance Immunity: An Emerging Paradigm of Innate Defense Activation in Caenorhabditis elegans. PLoS Pathog. 2016;12(9):e1005795.

61. Martineau CN, Kirienko NV, Pujol N. Innate immunity in C. elegans. Curr Top Dev Biol. 2021;144:309-51.

62. Pukkila-Worley R, Feinbaum R, Kirienko NV, Larkins-Ford J, Conery AL, Ausubel FM. Stimulation of Host Immune Defenses by a Small Molecule Protects C. elegans from Bacterial Infection. PLOS Genetics. 2012;8(6):e1002733.

63. Zachari M, Ktistakis N. Mammalian Mitophagosome Formation: A Focus on the Early Signals and Steps. Frontiers in cell and developmental biology. 2020;8.

64. McColl G, Roberts B, Pukala T, Kenche V, Roberts C, Link C, et al. Utility of an improved model of amyloid-beta $\left(A \beta_{1-42}\right)$ toxicity in Caenorhabditis elegans for drug screening for Alzheimer's disease. Molecular neurodegeneration. 2012;7.

65. Morley J, Brignull H, Weyers J, Morimoto R. The threshold for polyglutamine-expansion protein aggregation and cellular toxicity is dynamic and influenced by aging in Caenorhabditis elegans. Proceedings of the National Academy of Sciences of the United States of America. 2002;99(16).

66. Fan H, Ho L, Chi C, Chen S, Peng G, Chan T, et al. Polyglutamine (PolyQ) diseases: genetics to treatments. Cell transplantation. 2014;23(4-5):441-58.

67. Pi H, Xu S, Zhang L, Guo P, Li Y, Xie J, et al. Dynamin 1-like-dependent mitochondrial fission initiates overactive mitophagy in the hepatotoxicity of cadmium. Autophagy. 2013;9(11):1780-800.

68. Doxaki C, Palikaras K. Neuronal Mitophagy: Friend or Foe? Front Cell Dev Biol. 2020;8:611938.

69. Newton AP, Cadena SM, Rocha ME, Carnieri EG, Martinelli de Oliveira MB. Effect of triclosan (TRN) on energy-linked functions of rat liver mitochondria. Toxicol Lett. 2005;160(1):49-59.

70. Ajao C, Andersson MA, Teplova VV, Nagy S, Gahmberg CG, Andersson LC, et al. Mitochondrial toxicity of triclosan on mammalian cells. Toxicol Rep. 2015;2:624-37.

71. Shim J, Weatherly LM, Luc RH, Dorman MT, Neilson A, Ng R, et al. Triclosan is a mitochondrial uncoupler in live zebrafish. J Appl Toxicol. 2016;36(12):1662-7.

72. Teplova VV, Belosludtsev KN, Kruglov AG. Mechanism of triclosan toxicity: Mitochondrial dysfunction including complex II inhibition, superoxide release and uncoupling of oxidative phosphorylation. Toxicol Lett. 2017;275:108-17.

73. Weatherly LM, Shane HL, Friend SA, Lukomska E, Baur R, Anderson SE. Topical Application of the Antimicrobial Agent Triclosan Induces NLRP3 Inflammasome Activation and Mitochondrial Dysfunction. Toxicol Sci. 2020;176(1):147-61.

74. Weatherly LM, Gosse JA. Triclosan exposure, transformation, and human health effects. J Toxicol Environ Health B Crit Rev. 2017;20(8):447-69.

75. Dhillon GS, Kaur S, Pulicharla R, Brar SK, Cledon M, Verma M, et al. Triclosan: current status, occurrence, environmental risks and bioaccumulation potential. Int J Environ Res Public Health. 2015;12(5):5657-84.

76. MacDonald ML, Lamerdin J, Owens S, Keon BH, Bilter GK, Shang Z, et al. Identifying off-target effects and hidden phenotypes of drugs in human cells. Nat Chem Biol. 2006;2(6):329-37. 
77. Kumar P, Kumar A. Possible role of sertraline against 3-nitropropionic acid induced behavioral, oxidative stress and mitochondrial dysfunctions in rat brain. Prog Neuropsychopharmacol Biol Psychiatry. 2009;33(1):100-8.

78. Hwang HY, Shim JS, Kim D, Kwon HJ. Antidepressant drug sertraline modulates AMPK-MTOR signaling-mediated autophagy via targeting mitochondrial VDAC1 protein. Autophagy. 2020:1-17.

79. Duan W, Peng Q, Masuda N, Ford E, Tryggestad E, Ladenheim B, et al. Sertraline slows disease progression and increases neurogenesis in N171-82Q mouse model of Huntington's disease. Neurobiol Dis. 2008;30(3):312-22.

80. Peng $Q$, Masuda N, Jiang $M$, Li Q, Zhao $M$, Ross CA, et al. The antidepressant sertraline improves the phenotype, promotes neurogenesis and increases BDNF levels in the R6/2 Huntington's disease mouse model. Exp Neurol. 2008;210(1):154-63.

81. Gill JS, Jamwal S, Kumar P, Deshmukh R. Sertraline and venlafaxine improves motor performance and neurobehavioral deficit in quinolinic acid induced Huntington's like symptoms in rats: Possible neurotransmitters modulation. Pharmacol Rep. 2017;69(2):306-13.

82. Sharma N, Jamwal S, Kumar P. Beneficial effect of antidepressants against rotenone induced Parkinsonism like symptoms in rats. Pathophysiology. 2016;23(2):123-34.

83. Antonini A, Tesei S, Zecchinelli A, Barone P, De Gaspari D, Canesi M, et al. Randomized study of sertraline and low-dose amitriptyline in patients with Parkinson's disease and depression: effect on quality of life. Mov Disord. 2006;21(8):1119-22.

84. Kulisevsky J, Pagonabarraga J, Pascual-Sedano B, Gironell A, Garcia-Sanchez C, Martinez-Corral M. Motor changes during sertraline treatment in depressed patients with Parkinson's disease*. Eur J Neurol. 2008;15(9):953-9.

85. Tinsley JM, Fairclough RJ, Storer R, Wilkes FJ, Potter AC, Squire SE, et al. Daily treatment with SMTC1100, a novel small molecule utrophin upregulator, dramatically reduces the dystrophic symptoms in the mdx mouse. PLoS One. 2011;6(5):e19189.

86. Moore TM, Lin AJ, Strumwasser AR, Cory K, Whitney K, Ho T, et al. Mitochondrial Dysfunction Is an Early Consequence of Partial or Complete Dystrophin Loss in mdx Mice. Front Physiol. 2020;11:690.

87. Dubinin MV, Talanov EY, Tenkov KS, Starinets VS, Belosludtseva NV, Belosludtsev KN. The Effect of Deflazacort Treatment on the Functioning of Skeletal Muscle Mitochondria in Duchenne Muscular Dystrophy. Int J Mol Sci. 2020;21(22).

88. Stiernagle T. Maintenance of C. elegans. WormBook. 2006.

89. Benedetti C, Haynes CM, Yang Y, Harding HP, Ron D. Ubiquitin-like protein 5 positively regulates chaperone gene expression in the mitochondrial unfolded protein response. Genetics. 2006;174(1):22939.

90. Miedel M, Graf N, Stephen K, Long O, Pak S, Perlmutter D, et al. A pro-cathepsin L mutant is a luminal substrate for endoplasmic-reticulum-associated degradation in C. elegans. PloS one. 2012;7(7):e40145.

91. Link C, Johnson C. Reporter transgenes for study of oxidant stress in Caenorhabditis elegans. Methods in enzymology. 2002;353:497-505.

92. Bolz DD, Tenor JL, Aballay A. A Conserved PMK-1/p38 MAPK Is Required in Caenorhabditis elegans Tissue-specific Immune Response to $<i>$ Yersinia pestis</i> Infection. Journal of Biological Chemistry. 2010;285(14):10832-40.

93. Beanan MJ, Strome S. Characterization of a germ-line proliferation mutation in C. elegans. Development. 1992;116(3):755-66.

94. Lagido C, McLaggan D, Glover L. A Screenable In Vivo Assay for Mitochondrial Modulators Using Transgenic Bioluminescent Caenorhabditis elegans. Journal of visualized experiments : JoVE. 2015(105):e53083. 
95. Yang T, Zhao X, Zhang Y, Xie J, Zhou A. 6"'-Feruloylspinosin alleviated beta-amyloid induced toxicity by promoting mitophagy in Caenorhabditis elegans (GMC101) and PC12 cells. The Science of the total environment. 2020;715.

96. Pei J, Panina SB, Kirienko NV. An Automated Differential Nuclear Staining Assay for Accurate Determination of Mitocan Cytotoxicity. J Vis Exp. 2020(159).

\section{Figure Legends}

Figure 1. High-throughput, high-content PINK-1/PINK1 stabilization screen yielded eight hit compounds.

(a) The accumulation of PINK-1/PINK1 in the outer mitochondrial membrane of damaged mitochondria initiates mitophagy. 45,000 compounds were screened in C. elegans resulting in eight PINK-1/PINK1 stabilizers. (b) Fluorescent (Raw) and Cell Profiler-processed (Processed) images of $C$. elegans carrying Ppink-1::PINK-1/PINK1::GFP or N2 wild-type upon $24 \mathrm{~h}$ of treatment with $\mathrm{Na}_{2} \mathrm{SeO}_{3}, \mathrm{PS} 135$, or DMSO control. Representative images are shown. (c) Quantification of GFP fluorescence of $C$. elegans carrying Ppink-1::PINK-1/PINK1::GFP after $72 \mathrm{~h}$ treatment with $7 \mathrm{mM} \mathrm{Na}_{2} \mathrm{SeO}_{3}$ or DMSO control. Three biological replicates for (b, c) were performed and analyzed.

Figure 2. PS compounds induced mitochondrial fragmentation and mitophagy. (a, b) Fluorescent images and quantification of fluorescence of $C$. elegans carrying (a) Pmyo-3::GFP ${ }^{\mathrm{mt}}$ or (b) mCherry::LGG-1/LC3 upon $15 \mathrm{~h}$ of treatment with PS compounds. (c) Quantification of LysoTracker Red fluorescence upon 15 h of treatment with DMSO, PS compounds, CCCP, or rotenone. (d) Quantification of luminescence (normalized to GFP) of C. elegans carrying Psur-5::luciferase::GFP upon $19 \mathrm{~h}$ of treatment with DMSO, PS compounds, CCCP, or rotenone. For $(\mathbf{a}, \mathbf{b})$, percent distribution for each category was calculated and plotted, Chi-square statistic tests were performed, and representative images are shown. Three biological replicates with $\sim 30$ worms/replicate were analyzed. For $(\mathbf{c}, \mathbf{d})$, at least four biological replicates with $\sim 400$ worms/replicate were analyzed. $p$ values were determined from one-way ANOVA, followed by Dunnett's test. All fold changes were normalized to DMSO control (at $100 \%$ ). NS not significant, ${ }^{*} p<0.05,{ }^{* *} p<$ $0.01, * * * p<0.001$ 

consumption rate (pmol/min/worm) measurement of wild-type worms upon $8 \mathrm{~h}$ of treatment with PS compounds or rotenone. (b) Point plot of nonyl-acridine orange fluorescence (mitochondrial mass, $x$-axis) and MitoTracker Red (mitochondrial membrane potential, $y$-axis) fluorescence of wild-type worms upon $15 \mathrm{~h}$ of treatment with PS compounds or CCCP. (c) Quantification of DHE fluorescence upon $15 \mathrm{~h}$ of treatment with PS compounds or CCCP. At least three biological replicates with (a) 6,000 worms/replicate or (b, c) 400 worms/replicate were analyzed. $p$ values were determined from one-way ANOVA, followed by Dunnett's test. For $(\mathbf{b}, \mathbf{c})$, fold changes were normalized to DMSO control (at $100 \%)$. NS not significant, ${ }^{*} p<0.05, * * p<0.01, * * * p<0.001$. In (b), blue color indicates significance of changes in mitochondrial membrane potential; red indicates significance of changes in mitochondrial mass. represent SEM. $p$ values were determined from one-way ANOVA, followed by Dunnett's test. All fold 0.001 .

Figure 5. PS83 and PS106 reduced the rate of paralysis in C. elegans strain expressing human betaamyloid. (a-e) Rate of paralysis curve of C. elegans Alzheimer's model (GMC101) expressing full-length

735 human beta-amyloid upon treatment with (a, d) $5 \mu \mathrm{M}$ PS83, (b, e) $25 \mu \mathrm{M}$ PS106, or (c) 100 mM metformin 736 control. In (d, e), worms were reared on E. coli expressing $c d c-25.1(R N A i) / v e c t o r(R N A i)$ or $c d c-$ $73725.1(R N A i) / p i n k-1(R N A i)$. At least three biological replicates with 180 worms/replicate were analyzed. $p$ 
values were determined from Student's $t$-test. ${ }^{*} p<0.05,{ }^{* *} p<0.01,{ }^{* *} p<0.001$. Black stars indicate significance compared to DMSO control. Red stars in (d, e) indicated statistical significance between PS compound on vector vs pink-1(RNAi)-reared animals.

Figure 6. PS106 reduced aggregate formation in C. elegans strain expressing polyglutamine. (a, b)

Percent distribution of the number of polyglutamine aggregates in C. elegans strain expressing polyglutamine (Q82) (GF66) upon treatment with (a) $5 \mu \mathrm{M}$ PS83 or (b) $25 \mu \mathrm{M}$ PS106. Three biological replicates with $\sim 30$ worms/replicate were scored and analyzed. $p$ values were determined from Chisquare test. NS not significant, $* * * p<0.001$.

Figure 7. Most PS compounds have low toxicity in $C$. elegans and mammalian cells. Viability of (a) $C$. elegans, (b) human astroglial (SVG-P12) cell line, or (c) human prostate epithelial cell line (RWPE-1) upon 3 days of exposure to PS compounds with concentration gradient as indicated on the graphs. Dotted lines mark $75 \%$ of viability. At least three biological replicates were analyzed. Error bars represent SEM. All fold changes were normalized to DMSO control.

Table 1. Summary of PS compounds' effects on various mitochondrial parameters and other cellular pathways. NS not significant.

\section{Supporting Information}

754 Figure S1. Chemical structures of the eight PS compounds.

755 Figure S2. Three PS compounds reduced the rate of paralysis in C. elegans Alzheimer's model. Percent 756 paralysis of $C$. elegans GMC101 that expresses full-length human beta-amyloid upon treatment with (a-h) 
bioRxiv preprint doi: https://doi.org/10.1101/2021.06.07.447442; this version posted June 7, 2021. The copyright holder for this preprint (which was not certified by peer review) is the author/funder, who has granted bioRxiv a license to display the preprint in perpetuity. It is made available under aCC-BY 4.0 International license.

759 with $\sim 180$ worms/replicate were analyzed. $p$ values were determined from Student's $t$-test. NS not

760 significant, $* p<0.05, * * p<0.01, * * * p<0.001$

761 Table S1. Chemical information of the eight PS compounds.

762 Table S2. Tanimoto coefficient of the eight PS compounds as compared to each other. 


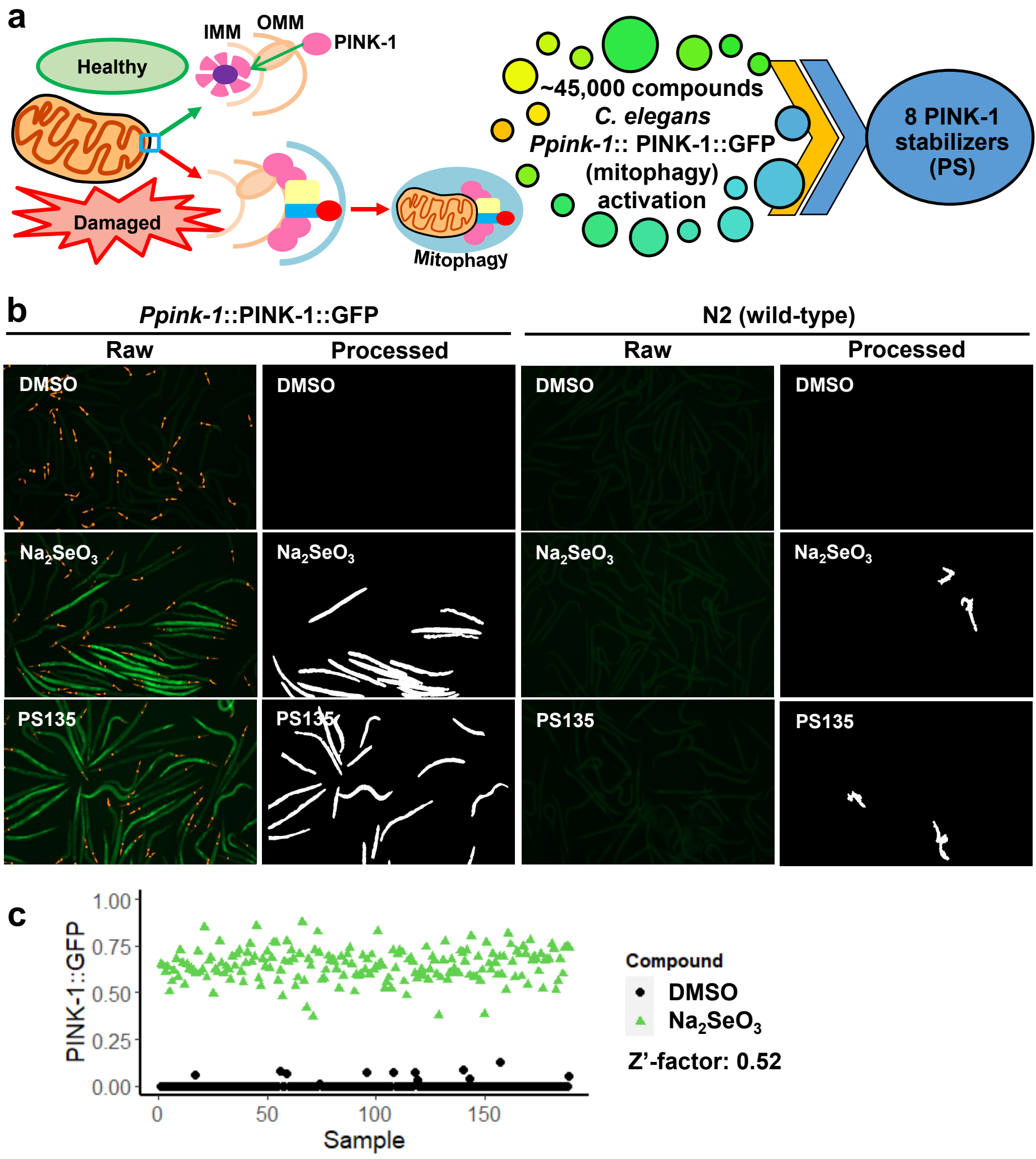

\section{Figure 1}




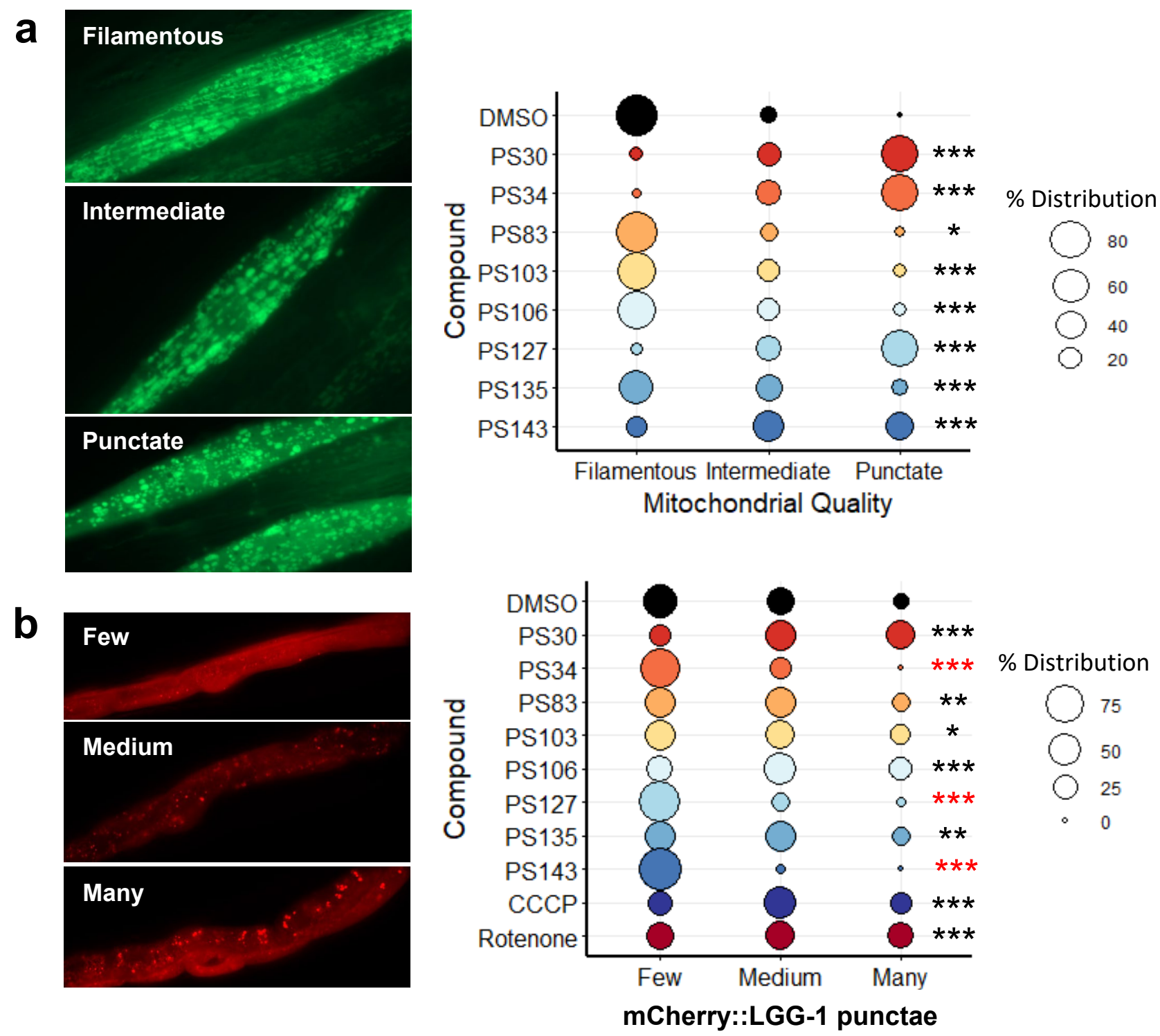

c
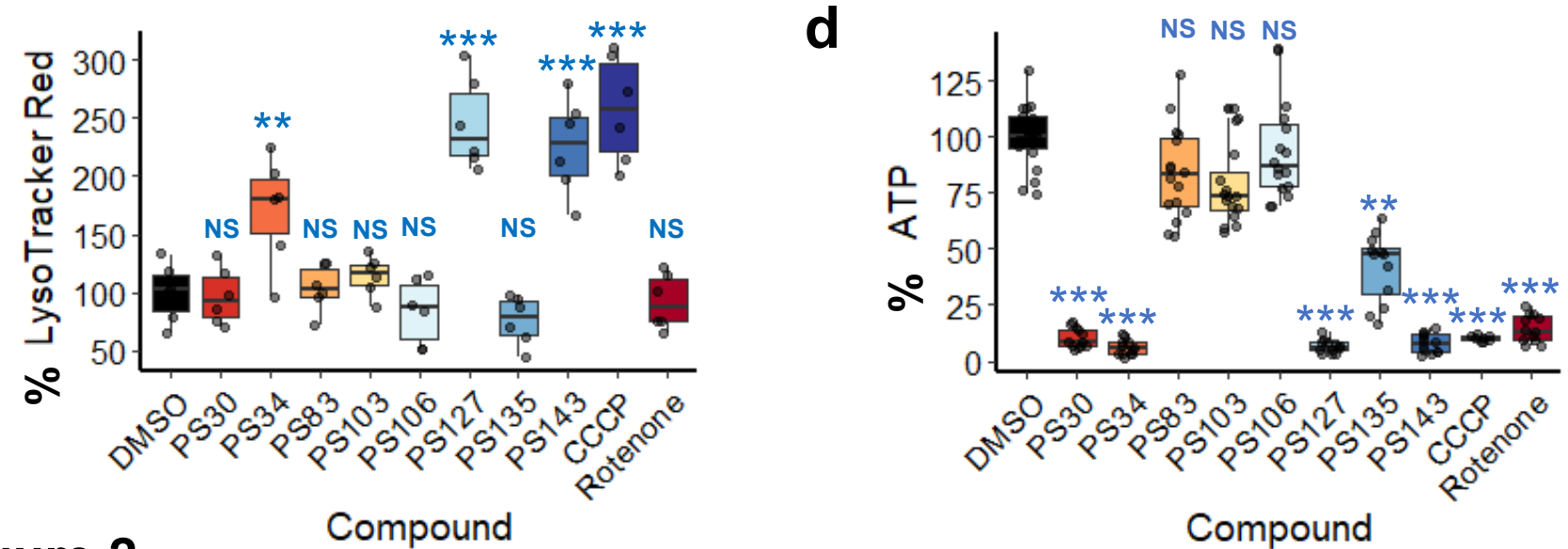

Figure 2

Compound 
a

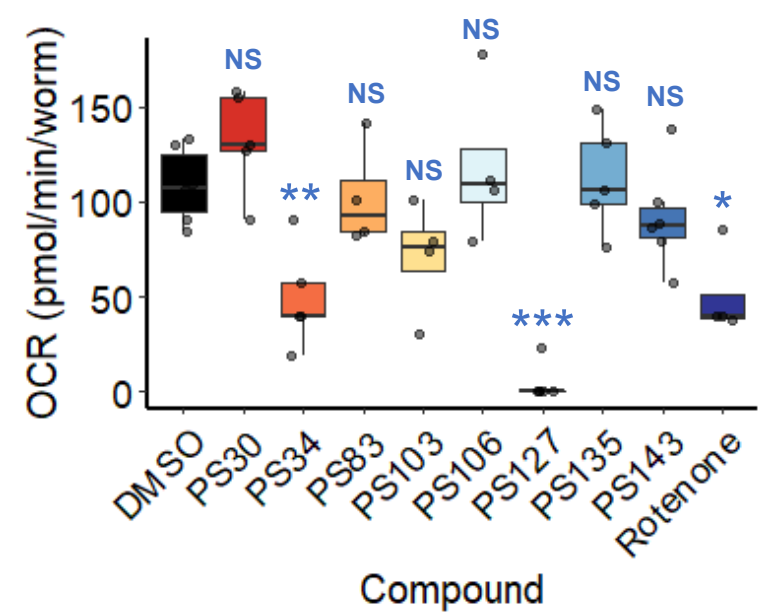

Compound
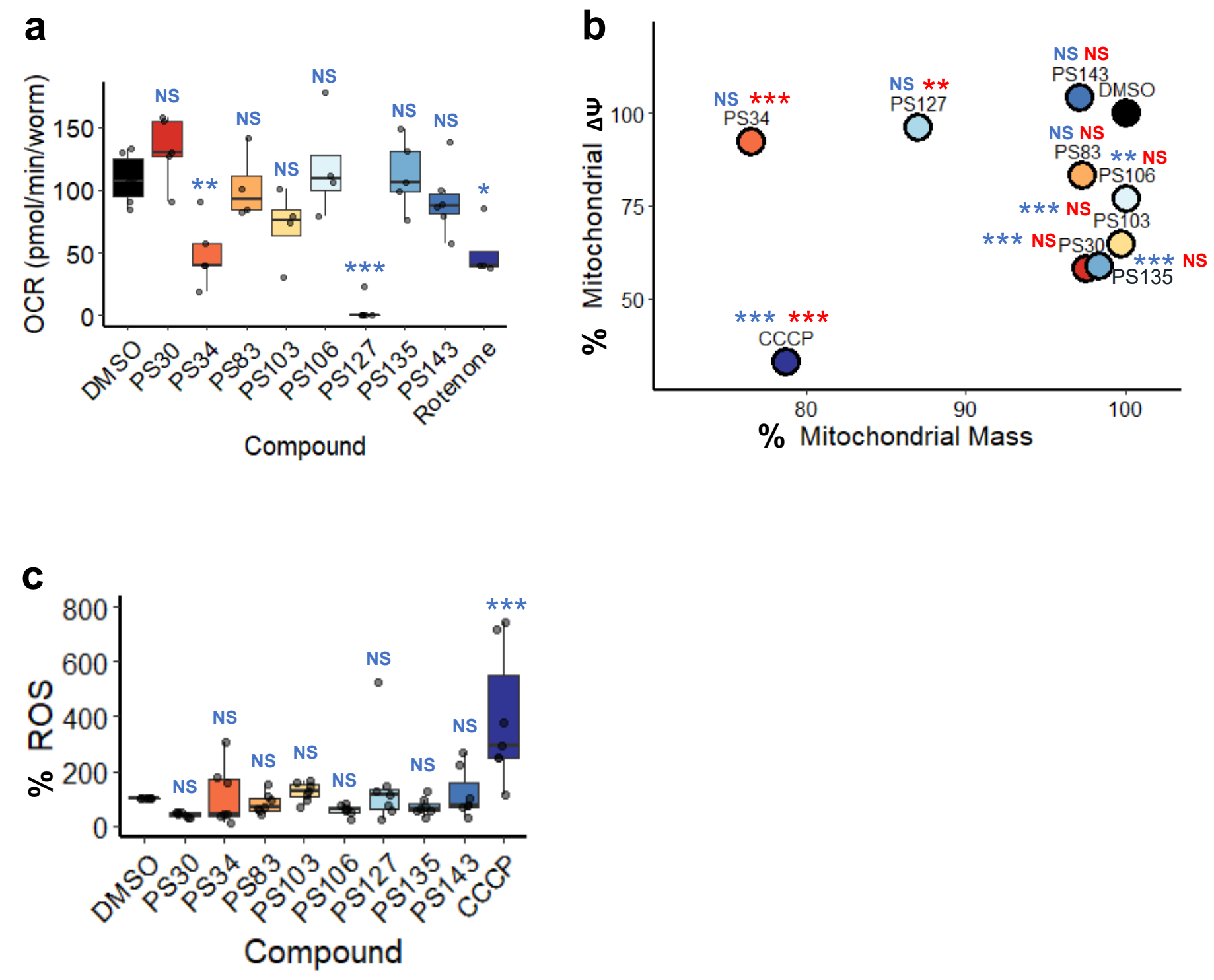

Figure 3 
a
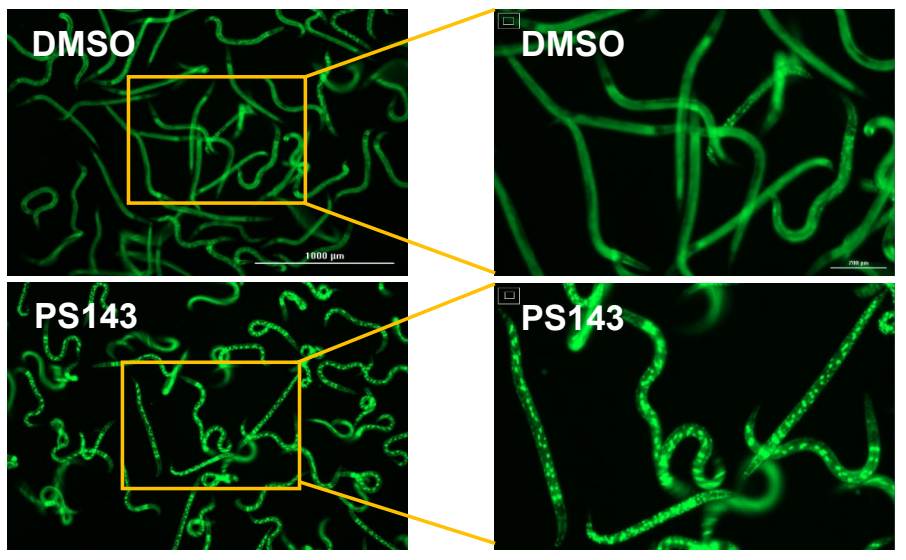

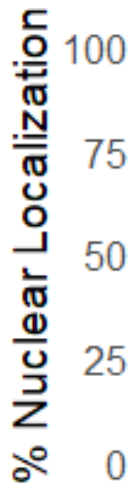

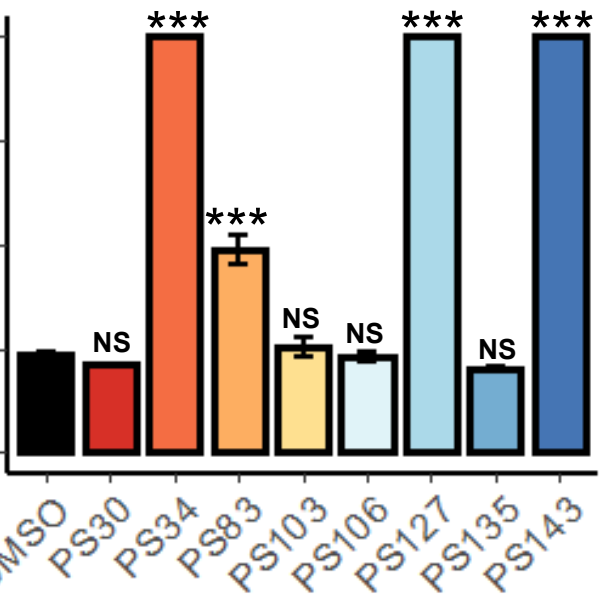

Compound

b

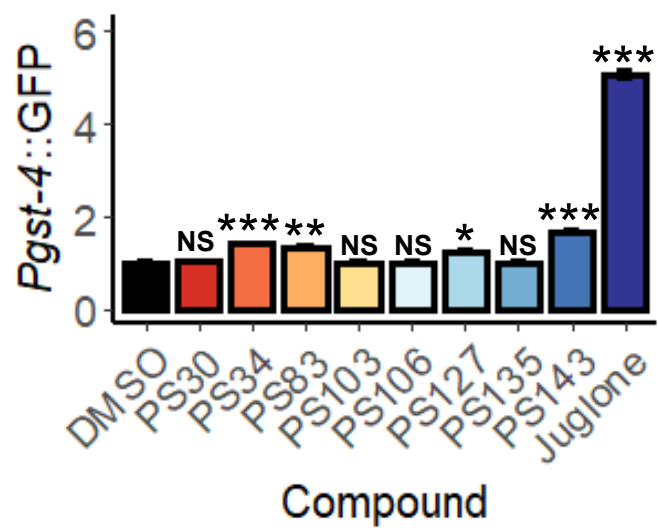

C

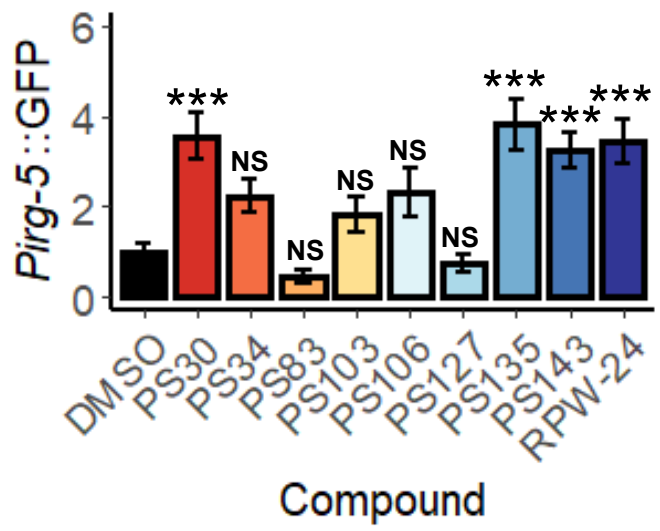

Figure 4 
a

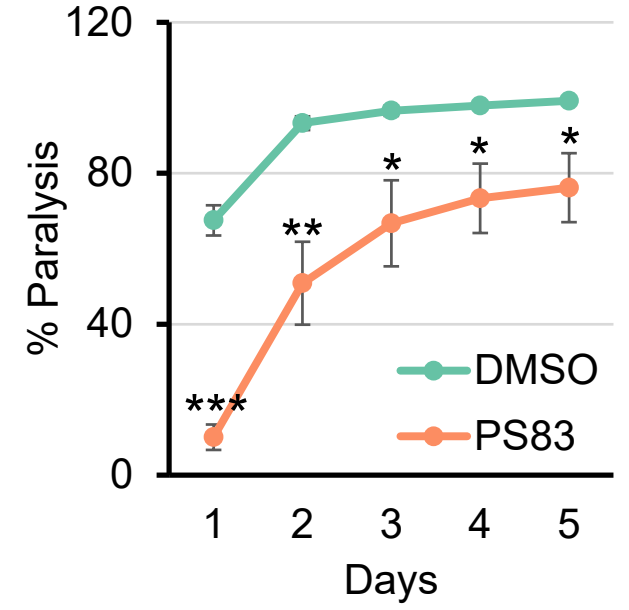

d

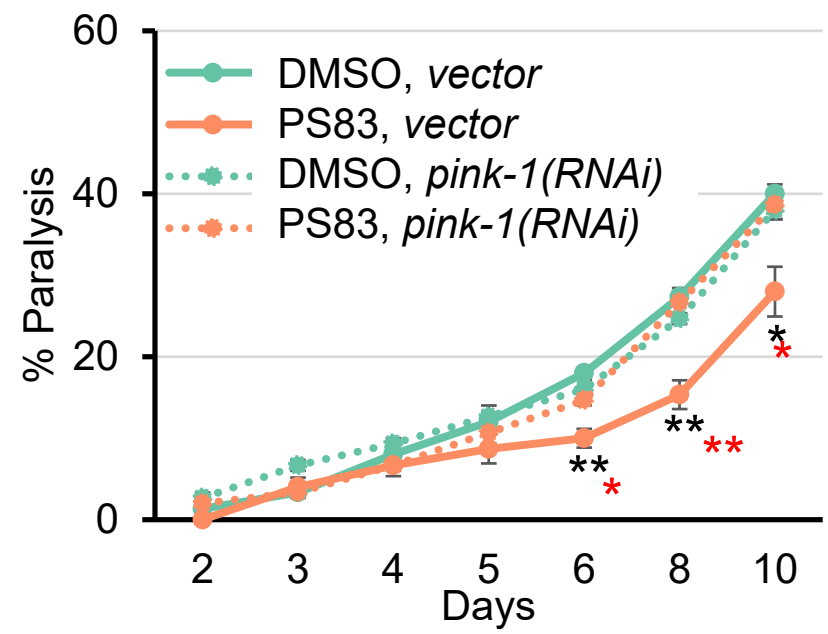

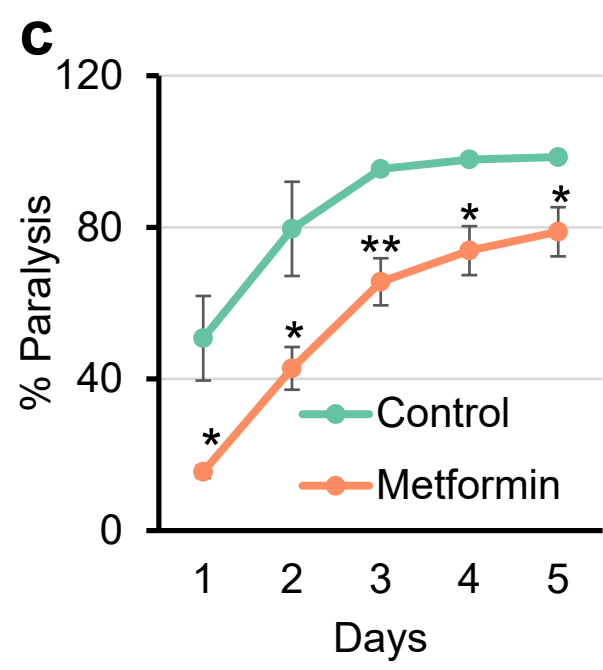

e

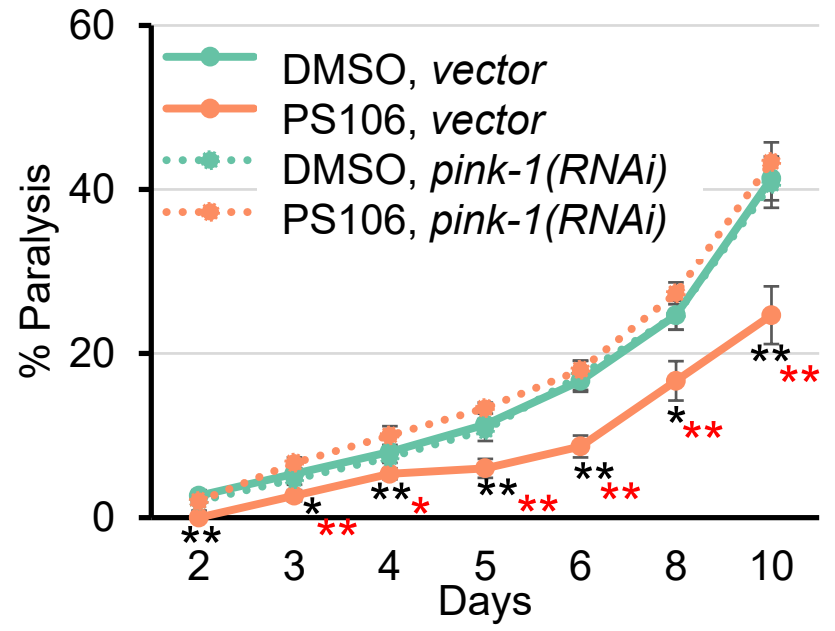

\section{Figure 5}


a

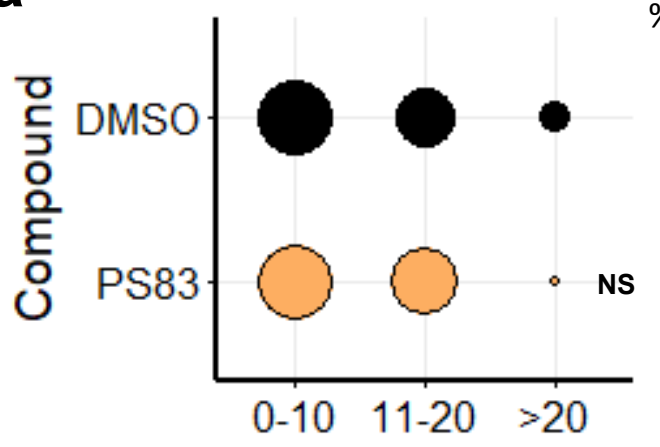

Number of Aggregates
\% Distribution

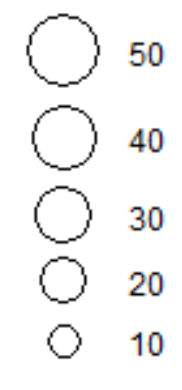

b

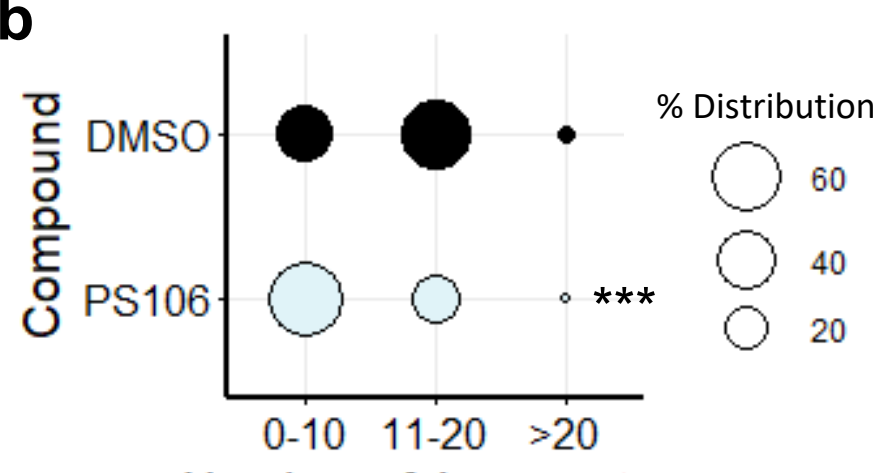

Number of Aggregates

Figure 6 
a

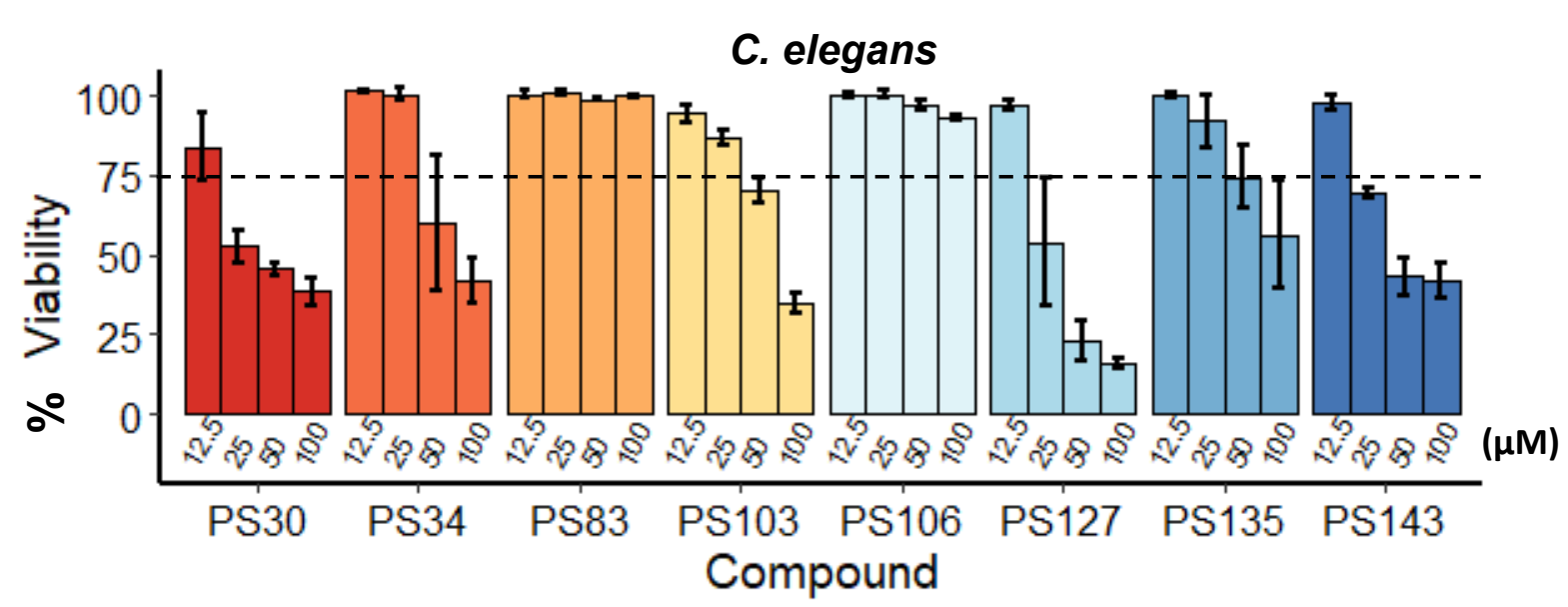

b
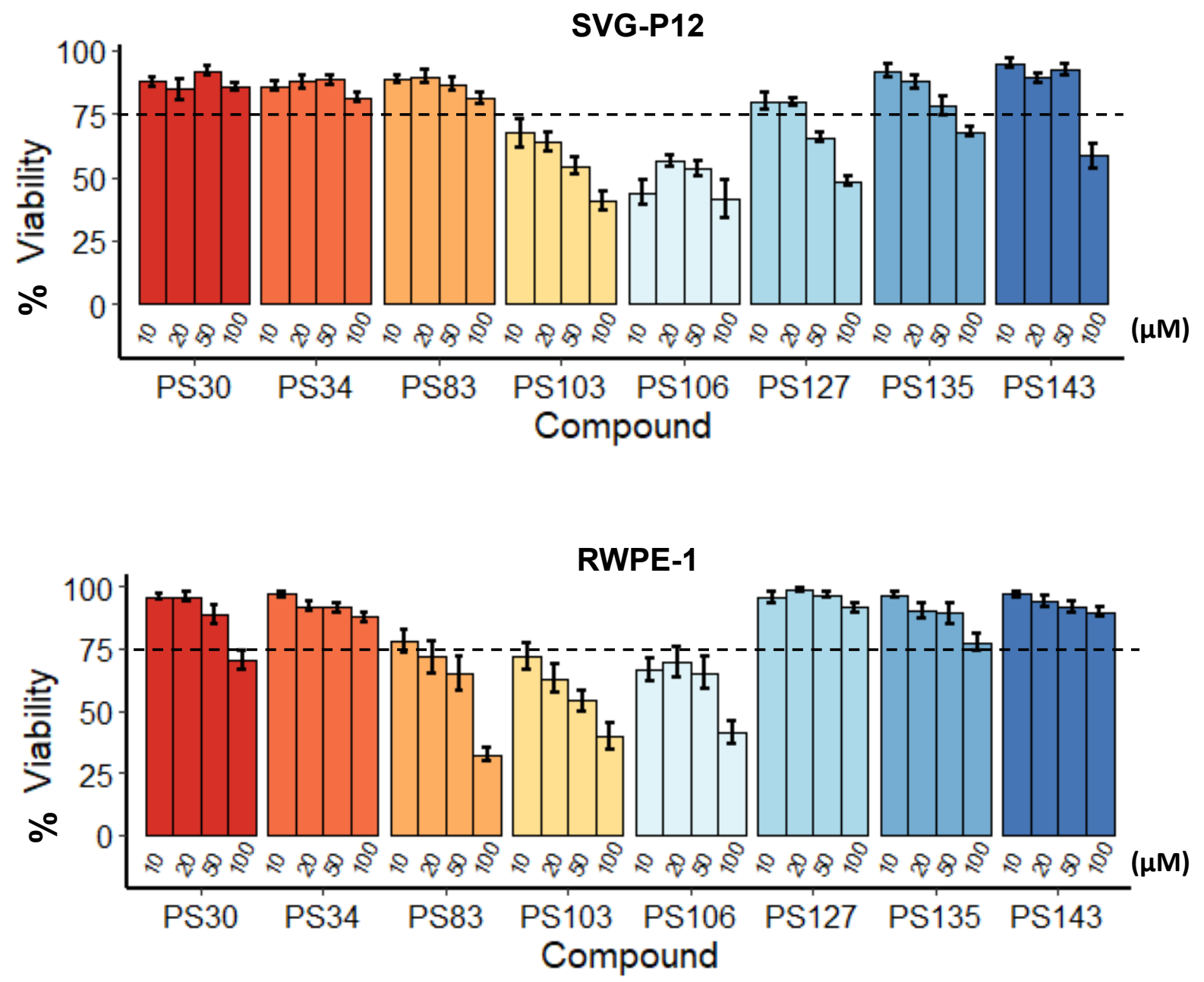

Figure 7 


\section{$\begin{array}{llllllll}\text { PS30 } & \text { PS34 } & \text { PS83 } & \text { PS103 } & \text { PS106 } & \text { PS127 } & \text { PS135 } & \text { PS143 }\end{array}$}

Mt fragmentation

Autophagosome formation

Autophagolysosome formation

ATP depletion

$\mathrm{O}_{2}$ consumption rate reduction

Mt mass reduction

Mt $\Delta \Psi$ reduction

ROS formation

GST-4 (SKN-1/Nrf2) induction

DAF-16/FOXO nuclear loc.

IRG-5 (immune response)

High High Low

High Less

Mid

Mid

Mid

Less

Mid

Less

NS High NS

NS

NS

High

NS

High

High High

NS

NS

NS

High

High

High

NS High NS

NS

NS

High

NS

NS

NS High NS

NS

NS

High

NS

NS

High NS

NS

High

Mid

NS

High

NS

NS

NS

NS

NS

NS

NS

NS

NS

NS Mid

Mid

NS

NS

Mid

NS

Mid

NS High High NS

NS

High

NS

High

High

NS NS

NS

NS

NS

High

High

\section{Table 1}

